\title{
The Genetics of Phenotypic Plasticity in Plant Defense: Trichome Production in Mimulus guttatus
}

\author{
Liza M. Holeski, ${ }^{\star}$ Ronnette Chase-Alone, ${ }^{\dagger}$ and John K. Kelly \\ Department of Ecology and Evolutionary Biology, University of Kansas, Lawrence, Kansas 66045 \\ Submitted August 6, 2009; Accepted December 9, 2009; Electronically published February 23, 2010 \\ Online enhancement: appendix. Dryad data: http://hdl.handle.net/10255/dryad.1127.
}

ABSTRACT: Insect herbivory is a major driving force of plant evolution. Phenotypic plasticity and developmental variation provide a means for plants to cope with variable herbivory. We characterized the genetics of developmental variation and phenotypic plasticity in trichome density, a putative defensive trait of Mimulus guttatus (yellow monkeyflower). Our results are evaluated in relation to the optimal defense theory, which provides testable predictions for plastic and developmental patterns in defense traits. We found that both developmental stage and simulated insect damage affected trichome production, but in different ways. Plants were more likely to produce at least some trichomes on later leaves than on earlier leaves, regardless of damage. Damage did not affect the average probability of producing trichomes, but it did increase the density of hairs on trichome-positive plants. We mapped trichome quantitative trait loci (QTL) by selectively genotyping a large panel of recombinant inbred lines derived from two highly divergent populations. Several highly pleiotropic QTL influenced multiple aspects of the trichome phenotype (constitutive, developmental, and/or plastic responses). Only one of the QTL influenced trichome induction following damage. In a result that is consistent with a central prediction of optimal defense theory, the high allele at this location was from the ancestral population with low constitutive trichome production.

Keywords: phenotype plasticity, Mimulus guttatus, QTL, trichomes, trade-off, optimal defense theory.

\section{Introduction}

As sessile organisms, plants must cope with variable herbivory while simultaneously allocating resources to growth and reproduction (e.g., Herms and Mattson 1992). Optimal defense theory (ODT) provides testable predictions for the evolution of plant resistance to herbivory (McKey 1974, 1979; Feeny 1975, 1976; Rhoades 1979). ODT is based on

\footnotetext{
* Corresponding author. Present address: Department of Entomology, University of Wisconsin, 1630 Linden Drive, Madison, Wisconsin 53706; e-mail: holeski@entomology.wisc.edu.

${ }^{+}$Present address: Environmental Science Program, College of Science, University of Texas, 500 West University, El Paso, Texas 79968.

Am. Nat. 2010. Vol. 175, pp. 391-400. (c) 2010 by The University of Chicago. 0003-0147/2010/17504-51506\$15.00. All rights reserved.

DOI: $10.1086 / 651300$
}

the proposition that defensive traits are costly because their production/maintenance draws resources from growth and reproduction. Stamp (2003) summarized the major features of this theory and outlined three basic predictions. First, plants evolve a level of defense that is positively related to attack rate by herbivores and negatively related to allocational or ecological cost. Second, plants will differentially allocate defense to different parts or tissues, with greater investment in tissues with high fitness values or where the cost of defense is lower. Third, plants will increase defense in response to attack, a form of plasticity that is often referred to as induction. ODT predicts that the capacity for induction should be negatively correlated with levels of constitutive defense.

ODT has been tested by direct evaluation of model predictions within natural populations. Intrapopulation studies provide support for the basic premises of ODT: herbivory is a strong selective agent (e.g., Marquis 1984; Mauricio and Rausher 1997), defensive traits deter herbivores (e.g., Berenbaum 1981; Simms and Rausher 1989), and resistance often carries significant allocational and/or ecological costs (Simms 1992; Bergelson and Purrington 1996; Purrington 2000; Strauss et al. 2002). An alternative approach is comparative: does ODT correctly predict differences among populations or species in defensive phenotypes? In this article, we couple the comparative approach with a detailed genetic dissection of interpopulation differences in plant defense.

The power of comparative studies is greatly increased where it is possible to cross divergent taxa. In hybrid genotypes, such as those in the $\mathrm{F}_{2}$ generation, the many trait differences between the parental genotypes will segregate more or less independently. The various effects of these different phenotypes on, for example, the rate of herbivore attack can then be distinguished (Jordan 1991; Nagy 1997; Lexer et al. 2003; Hall and Willis 2006; Wright et al. 2006). The extent to which different traits do not segregate independently is also essential information. Cosegregation of different phenotypes reflects a genetic correlation between 
these characters and is due to either pleiotropy or close linkage of the loci determining trait differences among taxa. The functional trade-offs inherent to ODT-and most other models in evolutionary ecology — should manifest as genetic correlations within a segregating hybrid population. When treating fitness within two environments as a pair of (potentially) correlated traits (e.g., chap. 19 in Falconer and Mackay 1996), ODT predicts a negative genetic correlation between plant fitness with herbivores and plant fitness without herbivores. This correlation should be evident in a cross between populations (genotypes) with high and low investments in defense.

Here we use mapping of quantitative trait loci (QTL) to identify specific genomic regions that contribute to variation in defense within a cross, and we compare our results with patterns predicted by ODT. While coarse-QTL routinely contain hundreds of genes-measuring phenotypic effects at the scale of genomic segments facilitates detection of evolutionary trade-offs (Kelly 2009). Here we asked whether QTL for different components of defense colocalized, that is, whether they mapped to the same genomic regions. In regard to ODT, we would like know the extent to which defense induction is controlled by the differential regulation of genes that are responsible for constitutive defense, as well as the extent to which juvenile and mature plant defenses are genetically and developmentally related. We are aware of only one study that has mapped QTL for constitutive and induced defenses: Kliebenstein et al. (2002) mapped QTL for constitutive and induced glucosinolate levels in Arabidopsis thaliana. Glucosinolates are a group of defensive secondary metabolites, and they were induced in response to methyl jasmonate treatment. QTL for trait induction colocalized with QTL for constitutive trait values (Kliebenstein et al. 2002). However, contrary to the expectations of ODT, constitutive and induced defenses were positively correlated.

In this article, we report the results of large-scale phenotypic and QTL analyses of ontogeny and plasticity of trichome production, a putative defense trait of Mimulus guttatus. Our mapping population was a large panel of recombinant inbred lines (RILs) derived from crossing two natural populations that are highly divergent for trichome density (Holeski 2007b). Several lines of evidence suggest that trichomes are a defensive trait in M. guttatus (see “Traits, Study Species, and Populations"). The Point Reyes population experiences relatively heavy herbivory, and its plants have high constitutive trichome densities (Holeski 2007a), whereas the Iron Mountain population experiences limited insect herbivory and its plants typically produce few trichomes. Our QTL mapping of trichome production directly tested ODT prediction that induction should be negatively correlated with constitutive defense.

\section{Material and Methods}

Traits, Study Species, and Populations

Trichomes are hairlike structures that extend from the epidermis and occur in a variety of types, including glandular or nonglandular and hooked or straight. They occur in a diversity of plant species and may serve a variety of defensive, physiological, and reproductive functions. For example, trichomes can reduce transpiration by reflecting light and by modifying the leaf boundary area conductance (Ehleringer 1984; Larcher 2001), they can increase tolerance to freezing, they accumulate and secrete excess salt (Gravano et al. 1998; Werker 2000), and they can facilitate wind dispersal of seed (Butterworth et al. 2009). Leaf trichomes have been shown to reduce insect herbivory in a number of species (Levin 1973; Marquis 1992; Agren and Schemske 1993; Mauricio 1998; van Dam and Hare 1998; Romeis et al. 1999; Malakar and Tingey 2000; Handley et al. 2005; Medeiros and Moreira 2005). They interfere with insect movement and feeding and often secrete glandular fluids that may trap, poison, or repel herbivores (Levin 1973; Elle and Hare 2000).

Yellow monkeyflower (Mimulus guttatus; Phrymaceae; Beardsley and Olmstead 2002) is a herbaceous plant that ranges from Mexico to Alaska in western North America and typically inhabits wet areas such as stream banks. Local populations differ extensively in morphology, life history (the species includes both annual and perennial ecotypes), mating system, biotic factors such as insect herbivory, and various abiotic conditions (Ritland 1989; Fenster and Ritland 1994; Kelly and Arathi 2003; Hall and Willis 2006; Hall et al. 2006; Holeski and Kelly 2006). Trichome density is highly variable in M. guttatus, and its trichomes are straight and unicellular and occur in the glandular form, often secreting a sticky and potentially noxious fluid. Constitutive trichome density is variable both within and among populations of M. guttatus, and much of this variation is genetic (Holeski 2007a).

Three lines of evidence suggest that trichomes in M. guttatus are an herbivory defense trait. First, constitutive trichome density has been shown to reduce insect herbivore damage on M. guttatus plants, although this effect may depend on the local assemblage of both herbivores and their predators (J. Haloin, unpublished data). Second, damage similar to insect herbivory on early leaves induces plants to produce higher trichome densities on later leaves (Holeski $2007 b$ ). This response is more clearly consistent with a defensive function than with alternative drought-related hypotheses. Third, consider the direction of differences between M. guttatus populations, both in phenotype and in the physical and biotic environments. Plants in our lowtrichome population at Iron Mountain, which is located in the Cascade Mountains of central Oregon, frequently pro- 
duce no or few trichomes. Iron Mountain plants have a short life span: only $\sim 2$ months elapse from bolting to death. Desiccation is the main cause of death. Iron Mountain plants typically experience little herbivory (Holeski 2007a). Our high-trichome population, Point Reyes National Seashore, is a perennial, low-elevation, streamside population located in the fog belt of coastal northern California. Plants from the Point Reyes population generally experience heavy herbivory (Holeski 2007a) but suffer little drought stress as a result of a relatively constant water supply. Also, the Iron Mountain population is subject to higher average solar radiation than is the Point Reyes population (US National Oceanic and Atmospheric Administration data for MayJuly, 1985-2005). In summary, the pattern of divergence between Iron Mountain and Point Reyes is opposite that predicted by the drought adaptation hypothesis: the drought-stressed population constitutively produces few trichomes.

\section{The Mapping Population}

We developed recombinant inbred lines (RILs) from a cross between an Iron Mountain plant (father) and a Point Reyes plant (mother). Iron Mountain 767 is a highly inbred line derived via single-seed descent, and it is typical of the Iron Mountain natural population in most of its measured phenotypic aspects (Scoville et al. 2009). The Point Reyes plant was an outbred plant that was two generations removed from the ancestral Point Reyes population, and it is representative of the natural population in all measured aspects of trichome production (L. M. Holeski, unpublished data). A single $F_{1}$ individual from the Iron Mountain $\times$ Point Reyes cross was self-fertilized to generate $\sim 1,000 \mathrm{~F}_{2}$ individuals, each of which then founded a distinct recombinant lineage. These lines were propagated through single-seed descent for four subsequent generations. Because of line loss, approximately 450 RILs remained in the $\mathrm{F}_{6}$ and $\mathrm{F}_{7}$ generations.

To achieve sufficient replication, we collected trichome data in three "measurement sets." In all cases, plants were grown in Fafard 3B potting soil (Conrad Fafard, Agawam, $\mathrm{MA}$ ) in 2-in pots in a greenhouse at either the University of Kansas (Lawrence) or the University of Wisconsin (Madison; 18-h day length; day/night temperatures, $25^{\circ}$ $\left.18^{\circ} \mathrm{C}\right)$. Plants were randomized within and among treatment categories and were rotated daily on the greenhouse bench. Plants were bottom-watered by flood irrigation for 1 h daily and were fertilized (Blossom Booster, J. R. Peters, Allentown, PA) once per week.

We counted trichomes on leaves from the second or fifth true leaf pair within a defined measuring area of 1 $\mathrm{cm}^{2}$ on the basal central part of the adaxial side of the leaf using a dissecting microscope $(4 \times$ magnification $)$. Tri- chome counts were performed on a particular leaf as soon as the succeeding leaf pair (third or sixth) was fully expanded (Holeski 2007b). This ensured that measurements were taken at the same particular development stage across plants (Orians 2005; Morris et al. 2006). This practice also effectively controls for leaf area, since leaves harvested at this stage are very similar across plants. Also relevant, we found that damage to second leaves does not affect area of fifth leaves (L. M. Holeski, unpublished data). Thus, any damage effects on trichome density are not due to alteration of leaf expansion.

Estimating trichome density from counts within a defined portion of a leaf is standard practice (e.g., Agrawal 2004; Mauricio 2005; Symonds et al. 2005; Traw and Feeny 2008). Trichome counts from within the region we used are strongly correlated with trichome counts from the leaf tip (A. Scoville, unpublished data) and should thus be a good proxy for the whole-leaf trichome number. In herbaceous plants, the second true leaf pair is in the juvenile developmental stage and the fifth leaf pair is generally considered to be in the adult or "mature" stage (Poethig 1997; Karban and Thaler 1999). We used fifth-leaf trichome counts to assess induction (and the corresponding constitutive values) because a preliminary experiment showed that induction was more pronounced in fifth leaves than in later (seventh) leaves (L. M. Holeski, unpublished data).

All trichome counts on second leaves were performed on undamaged plants. Fifth-leaf counts were performed on plants with and without damage to earlier leaves. For the former, we simulated insect-chewing damage by punching two holes per leaf, each $\sim 6 \mathrm{~mm}$ in diameter, in both leaves of the second leaf pair (Holeski 2007b). Measurement sets differed slightly in detail. In set 1 , we measured constitutive second-leaf trichome density as well as fifth-leaf induced trichome density of $\mathrm{F}_{6}$ plants. In set 2, we measured induced and uninduced fifth-leaf trichome density of $\mathrm{F}_{6}$ plants using a discrete scoring system $(0,1$, or $>1$ trichomes $\left.\mathrm{cm}^{-2}\right)$. In set 3 , we assessed induced and uninduced fifth-leaf trichome density of $\mathrm{F}_{7}$ plants by averaging counts from both leaves of the fifth leaf pair to determine whether this would reduce estimation error.

In aggregate, we have RIL mean estimates for three distinct phenotypes: (1) constitutive second-leaf trichome counts (435 RILs; 1,632 plants), (2) constitutive fifth-leaf trichome counts (211 RILs; 1,126 plants, 315 of which had both fifth leaves evaluated), and (3) induced fifth-leaf trichome counts (441 RILs; 2,709 plants, 311 of which had both fifth leaves evaluated). We used Minitab 14 (Minitab, State College, PA) and JMP 7 (SAS Institute, Cary, NC) for statistical analyses of data to address three specific questions: (1) How does developmental stage and/or simulated insect damage affect trichomes? (2) Is there genetic variation and/ 
or genotype $\times$ environment variation for either the probability of producing trichomes or trichome density? And (3) are there genetic correlations between trichome production traits? If so, what are their direction and magnitude?

Trichome number per leaf was highly right-skewed (fig. 1). We partitioned trait variation into two components, first by classifying plants dichotomously as trichome positive (1) or trichome negative (0) and then by evaluating variation in density among trichome-positive plants. These counts were log-transformed for normality. Our procedure is analogous to partitioning total fitness into survivorship and then fecundity among survivors. We applied a logit analysis (logistic regression with categorical predictors) to determine whether there was genetic variation for probability of producing trichomes. Measurement set, RIL, and damage treatment were included as categorical predictors, and we allowed an interaction between RIL and damage treatment.

Subsequent analyses, including the QTL mapping study, were based on RIL means. For each RIL, we determined the fraction of plants with at least one trichome on the second and fifth leaf pair, respectively. "Trichome2" is the arcsin-square-root-transformed proportion for second leaves (Sokal and Rohlf 2000, pp. 419-422). "Trichome5" is the corresponding value for fifth leaves. Constitutive trichome density is the log-transformed count from trichome-positive, undamaged plants. Induced trichome density is the difference between the log-transformed counts of damaged and undamaged plants within a RIL. Like constitutive trichome density, induced trichome density was based entirely on trichome-positive plants. For all four statistics, we calculated the least squares means for each RIL, correcting for slight differences among measurement sets. We assessed genetic correlations between trichome production traits by calculating Pearson productmoment coefficients.

\section{QTL Mapping}

The genotyping was performed in three stages. We first performed a preliminary screen on a set of approximately 500 Mimulus gene-based markers (derived from expressed sequence tags). This screen identified 163 informative markers (length polymorphic between the Iron Mountain and the Point Reyes parents) spanning the 14 linkage groups of M. guttatus. We then genotyped these markers in 47 RILs that were chosen to represent the range of trichome phenotypes. This is the selective genotyping approach (Darvasi 1997; Lynch and Walsh 1998). With genotypes for the 47 RILs, we conducted a single-marker analysis for each trichome trait, using generalized least squares to account for differences among RILs in the accuracy of mean phenotype estimates. This single-marker analysis was a weighted linear regression of trait values onto number of Point Reyes alleles

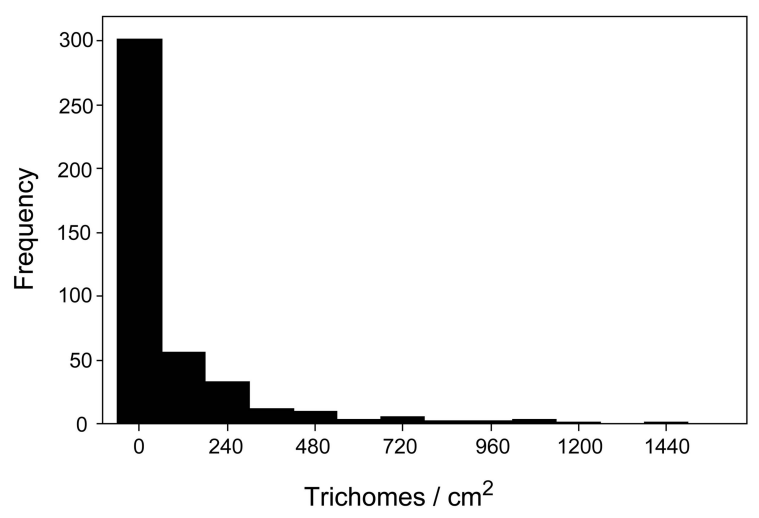

Figure 1: The frequency distribution is given for recombinant inbred line means of trichomes per square centimeter. This figure pools constitutive second and fifth leaf trichome counts from undamaged plants.

$(0,1$, or 2$)$. The third stage of genotyping was limited to 40 markers in the nine genomic regions with suggested effects on one or more of the trichome phenotypes (see fig. A1 in the online edition of the American Naturalist). Using these markers, we screened a total of 234 RILs with phenotypic data (the 47 RILs of the selective genotyping round are a subset of this collection of 234 RILs).

Using genetic data from the full screen, we constructed linkage maps (fig. A1) with the program AntMap, version 1.2 (Iwata and Ninomiya 2006). Generally, marker positions on our genetic linkage maps corresponded with the placement in previous maps (e.g., Hall and Willis 2005; Lee 2009). We mapped QTL using the composite interval mapping (CIM) function of Windows QTL Cartographer 2.0 (Wang et al. 2007). For CIM, we used the default model settings: the forward regression method with five control markers, a window size of $10 \mathrm{cM}$, and a walk speed of $2 \mathrm{cM}$. Genomewide threshold values were established for each trait (at significance level of $P \leq .05)$ using 300 permutations of the phenotypes against the genotypes (Churchill and Doerge 1994; Wang et al. 2007). The indicated significance thresholds were similar across traits: Critical logarithm (base 10) of odds $=2.77$ for trichome2, 2.91 for trichome5, 3.04 for constitutive trichome density, and 3.34 for induced trichome density. We used the QTL results to address four main questions: (1) What is the genetic architecture of constitutive trichome production? (2) Does the genetic architecture of constitutive trichome density change with ontogeny? (3) What is the genetic basis of trichome induction? And (4) what is the relationship between QTL for constitutive and induced trichome production?

\section{Results}

Across all plants, we found that $41.5 \%$ of second-leaf counts were trichome positive $(n=1,632), 66.0 \%$ of fifth- 
leaf counts from nondamaged plants were positive $(n=$ $1,126)$, and $67.6 \%$ of fifth-leaf counts from damaged plants were positive $(n=2,709)$. Developmental stage significantly affected the probability of producing trichomes, but damage did not (table 1). In contrast, developmental stage did not affect the density of trichomes produced in trichome-positive plants; second-leaf and fifth-leaf constitutive trichome densities did not significantly differ $\left(F_{1,1,028}<0.001 ; P=.99\right)$. Damage had a significant effect on fifth-leaf trichome density, as damaged plants had significantly higher fifth-leaf trichome densities than did undamaged plants $\left(F_{1,2,902}=23.05 ; P<.001\right)$.

The logit analysis demonstrated significant genetic variation and genotype $\times$ environment $(\mathrm{G} \times \mathrm{E})$ interaction for both the probability of producing trichomes and trichome density (table 2). Our standard ANOVA (normal residuals) indicated significant effects of damage, RIL, and a RIL $\times$ damage interaction on trichome density among trichome-positive plants (table 2). There were significant genetic (among-RIL) correlations between all four traits. Among trichome-positive plants, RIL explained about 50\% of the variance in trichome density within both undamaged and damaged plants.

Genetic correlations can be estimated from RIL means. The probability of producing trichomes on the second leaf pair (trichome2) was positively correlated with the probability of producing trichomes on the fifth leaf pair (trichome5; $r=0.36, P<.001)$ and with constitutive trichome density $(r=0.42, \quad P<.001)$. Trichome2 was negatively correlated with induced trichome density $(r=-0.19, P<.02)$. Trichome5 was positively correlated with constitutive trichome density $(r=0.22, P<.01)$ and induced trichome density $(r=0.16, P<.05)$.

Most relevant to ODT is the relationship between constitutive and induced trichome densities. If constitutive trichome production is unrelated to induction, then the

Table 1: Number of plants with trichomes absent or present for each measurement set

\begin{tabular}{|c|c|c|c|c|}
\hline \multirow{2}{*}{$\begin{array}{l}\text { Measurement set, } \\
\text { leaf pair }\end{array}$} & \multicolumn{2}{|c|}{ Trichomes } & \multirow[b]{2}{*}{$\chi^{2}$} & \multirow[b]{2}{*}{$P$} \\
\hline & Absent & Present & & \\
\hline 1 & & & 196.36 & $<.01$ \\
\hline 2nd leaf & 954 & 678 & & \\
\hline 5th leaf (damage) & 544 & 1,061 & & \\
\hline 2 & & & 2.29 & .13 \\
\hline $\begin{array}{l}\text { 5th leaf (no } \\
\text { damage) } \\
\text { 5th leaf (damage) }\end{array}$ & $\begin{array}{l}313 \\
275\end{array}$ & $\begin{array}{l}352 \\
366\end{array}$ & & \\
\hline 3 & & & 1.31 & .25 \\
\hline $\begin{array}{l}\text { 5th leaf (no } \\
\text { damage) }\end{array}$ & $\begin{array}{l}177 \\
158\end{array}$ & $\begin{array}{l}599 \\
616\end{array}$ & & \\
\hline
\end{tabular}

Note: Parts 1, 2, and 3 refer to measurement sets 1,2, and 3 respectively.
Table 2: Summary of the results of a logit analysis with the probability of producing fifth-leaf trichomes as the response variable and of an ANOVA with log fifth-leaf trichome density per square centimeter as the response variable

\begin{tabular}{lrcr}
\hline Analysis, source of variation & $\mathrm{df}$ & $\mathrm{L}-\mathrm{R} \chi^{2}$ & $P$ \\
\hline $\begin{array}{l}\text { Probability of producing } \\
\quad \text { trichomes: }\end{array}$ & & & \\
Measurement set & 2 & 272.982 & $<.001$ \\
RIL & 209 & $1,666.92$ & $<.001$ \\
Damage & 1 & .00053 & .982 \\
RIL $\times$ damage & 209 & 281.790 & $<.001$ \\
Density among trichome-positive & & & \\
$\quad$ plants: & & & \\
Measurement set & 1 & 2.12 & .145 \\
RIL & 299 & 2.32 & $<.001$ \\
Damage & 1 & 23.86 & $<.001$ \\
RIL $\times$ damage & 299 & 2.73 & $<.001$ \\
Error & 1,617 & & \\
\hline
\end{tabular}

Note: For the logit analysis with the probability of producing fifthleaf trichomes, the score for each plant/leaf pair is either 0 or 1 . The test statistic for logit $\left(\mathrm{L}-\mathrm{R} \chi^{2}\right)$ is a likelihood ratio evaluated relative the $\chi^{2}$ distribution. Recombinant inbred line (RIL) is a random factor in the ANOVA, all other factors are fixed, and $F$ is the test statistic. Both statistical tests include only those RILs with replicates that had and had not received second-leaf damage

slope of the regression of RIL mean trichome count when damaged onto mean trichome count when undamaged should equal 1 . While the relationship between counts is positive (fig. 2), the slope is much less than 1 , indicating that the induction response decreases in magnitude as the constitutive trichome density increases. In other words, there is a negative genetic correlation between constitutive trichome density and induction. Estimation error in the predictor variable ( $X=$ constitutive counts) biases slope estimates downward in linear regression, but that bias can be corrected with an estimate for the reliability ratio (Fuller 1987, chap. 1). Given RIL-specific sample sizes and the variance partitioning from a one-way ANOVA, we obtained a reliability ratio of 0.84 . The bias-corrected slope is $0.322(\mathrm{SE}=0.056)$, far below a value of 1 .

Of the nine regions suggested by our initial screen of 47 RILs, only four QTL (on linkage groups [LGs] 2, 5, 10, and 14) had significant effects on constitutive, ontogenetic, and/ or induced patterns of trichome production when analyzing the full RIL set (table 3; see also fig. A2 in the online edition of the American Naturalist). Two of these QTLs affected a single trait while the other two influenced multiple aspects of trichome production. Assuming that the QTLs combined additively, they cumulatively explained $27 \%$ of the variance among RIL means for trichome2, 53\% for trichome5, 41\% for constitutive trichome density, and $16 \%$ for induced trichome density (table 3 ). We did not have extensive replication of the two parental lines in the present experiment, 


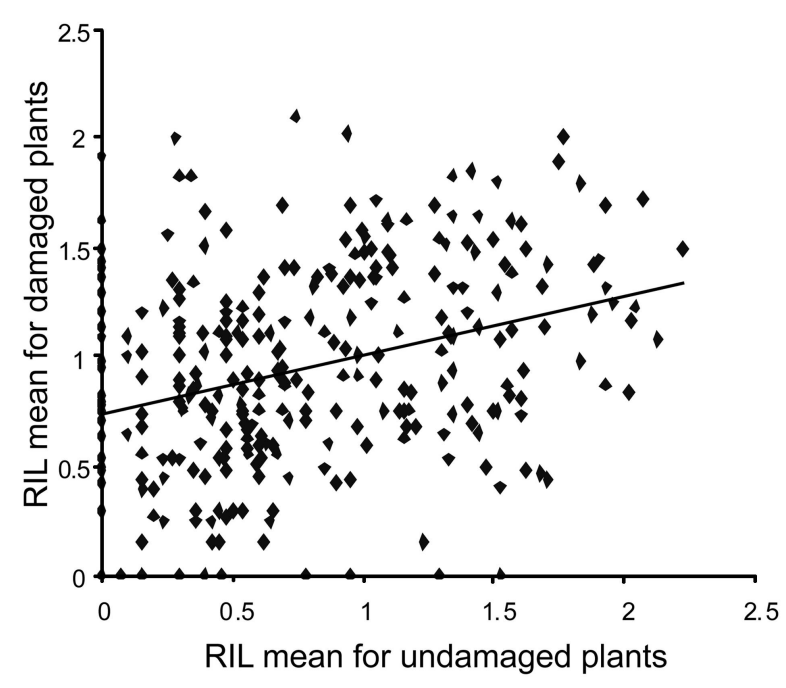

Figure 2: The relationship between log-transformed counts for damaged and undamaged plants (trichome-positive plants) across RILs is described. The estimated (unadjusted) regression equation is given by the solid line $y=0.737+0.271 x$ (see text for bias correction).

but previous studies indicated that values for trichome 2 and trichome 5 are close to 0 for the Iron Mountain population and close to 1 for the Point Reyes population (Holeski 2007a). Using these estimates, our QTL explained about $50 \%$ of the divergence between the Point Reyes and the Iron Mountain parents in trichome 2 and $75 \%$ of the divergence in trichome5. Constitutive trichome density and induced trichome density exhibited transgressive segregation; that is, the RIL population exhibited values outside the range of the parents. Thus, we report only the percent variance in the RIL population explained by a particular QTL for these traits.

The pleiotropic QTL (LGs 10 and 14) had the largest effects. The allelic effects were typically in the direction expected, given the parental line phenotypes: the Point Reyes allele increased trait values. There were, however, two exceptions. First, the Point Reyes allele on LG 2 reduced the likelihood of producing second-leaf trichomes, partially offsetting the large positive QTLs on LGs 10 and 14. Second, for the only QTL that significantly influenced induced trichome density, the Iron Mountain allele had a positive effect.

\section{Discussion}

Inducible defenses are routinely found in organisms under selective pressure from predators, parasites, herbivores, and pathogens (Harvell and Tollrian 1999). Optimal defense theory (ODT) predicts a negative correlation be- tween constitutive defense and induced defense. We tested this prediction using a cross between the divergent Iron Mountain and Point Reyes populations followed by quantitative trait locus (QTL) mapping of recombinant inbred lines (RILs). Within the RIL population, significant genetic correlations existed between trichome production at different developmental stages and between constitutive defense and induced defense. The mapping study documents perhaps the first example of a QTL-specific trade-off between constitutive defense and induced defense, consistent with a central prediction of ODT.

The Iron Mountain and Point Reyes populations were chosen for this study because they span the range for constitutive trichome production: low for Iron Mountain and high for Point Reyes. ODT predicts that the Iron Mountain population, which typically experiences relatively low herbivory and has a very short interval in which to grow and reproduce, should have low constitutive defenses and may induce defenses as needed. In contrast, the Point Reyes population typically experiences relatively high herbivory and has a longer growing season. ODT predicts higher levels of constitutive defense and a reduced need for induction. Three of our results directly supported these predictions: (1) in the RIL population, constitutive trichome density and induced trichome density were significantly negatively genetically correlated; (2) the QTL on LG 14 exhibited a tradeoff for constitutive trichome density and induced trichome density; and (3) in terms of the direction of the QTL-level trade-off, the Point Reyes allele at this QTL increased constitutive trichome density while the Iron Mountain allele increased induced trichome density.

\section{Variation in the Trichome Phenotype}

In the two developmental stages examined in our study, plant developmental stage but not damage significantly increased the probability of producing trichomes (table 1 ). In contrast, damage but not developmental stage significantly increased the density of trichomes produced in trichome-positive plants. We found significant genetic variation for both traits (table 2). Inter-RIL variation reflects the genetic divergence between the Iron Mountain and the Point Reyes populations. The large sample sizes in this study allowed us to detect two novel patterns that were evident only after separating presence/absence of trichomes from trichome density. First, we detected a significant RIL $\times$ damage interaction for both the probability of producing trichomes and trichome density (table 2; nonsignificant in Holeski 2007b). Second, we determined that developmental stage affected the probability of producing trichomes. However, among trichome-positive plants, average constitutive trichome density did not differ between the second and the fifth leaf pairs. 
Table 3: The linkage group and nearest marker(s) for each significant quantitative trait locus

\begin{tabular}{lccccc}
\hline & & \multicolumn{3}{c}{ Trait } \\
\cline { 3 - 6 } Analysis, linkage group & $\begin{array}{c}\text { Nearest } \\
\text { marker(s) }\end{array}$ & Trichome2 & Trichome5 & $\begin{array}{c}\text { Constitutive trichome } \\
\text { density }\end{array}$ & Induced trichome density \\
\hline Standardized effects $(a):$ & & & & & \\
2 & 513,761 & -.221 & $\ldots$ & $\ldots$ & $\ldots$ \\
5 & 641 & $\ldots$ & .176 & $\ldots$ & $\ldots$ \\
10 & 519,846 & .323 & .706 & .414 & $\ldots$ \\
14 & 137 & .348 & $\ldots$ & .486 & \\
Proportion of RIL & & & & & $\ldots$ \\
genetic variance $\left(a^{2}\right):$ & & & & $\ldots$ & $\ldots$ \\
2 & 513,761 & .049 & $\ldots$ & .396 & $\ldots$ \\
5 & 641 & $\ldots$ & .031 & .171 & .157 \\
10 & 519,846 & .104 & .499 & .237 & .16 \\
14 & 137 & .121 & $\ldots$ & .41 & $\ldots$ \\
\hline
\end{tabular}

Note: Standardized effects $(a)$ are the standardized additive effects of the Point Reyes allele at each QTL (in phenotypic SD). Data for trichome2 are the arcsin-square-root-transformed proportions for second leaves; for trichome 5 they are the corresponding values for fifth leaves. Data for constitutive trichome density are the log-transformed counts from trichome-positive, undamaged plants; for induced trichome density, they are the differences between the log-transformed counts of damaged and undamaged, trichome-positive plants within a recombinant inbred line (RIL).

Our results suggest that whether a leaf produces any trichomes is more developmentally canalized than is trichome density. The plasticity versus canalization of patterns of trichome production could have both ecological and evolutionary effects (DeWitt et al. 1998; Karban et al. 1999). If trichome production is costly, plants with a canalized trichome-negative ontogenetic trajectory may be less suited to defend themselves against herbivores, but they are more resource efficient when herbivores are absent. In trichomepositive plants with increased trichome density following herbivory, developmental variation and induction could be complementary strategies to maximize resistance to herbivory while minimizing potential costs of trichome production.

RILs differed in their responses to simulated herbivory (significant RIL $\times$ damage interactions; table 2). Depending on the precision of Mimulus guttatus's signal-andresponse mechanism for induction, genotypic response could vary with the type of damage received. For example, in plants with precise signaling networks, damage by chewing herbivores versus by sucking herbivores can elicit different plant-induced responses; certain genotypes might induce in response to one of these forms of herbivory but not the other (Karban and Baldwin 1997). Thus, the genotype $x$ environment interaction for induction indicates that particular genotypes could have increases and/or decreases in fitness (relative to other genotypes in the population) as associated herbivore populations increase or decrease in abundance.

Our use of simulated insect damage in these experiments has both advantages and disadvantages. The main disad- vantage is that the experimental results may not be directly applicable to any particular natural enemy, depending on the specificity of the induction response to cues from the herbivore. However, M. guttatus is attacked by a number of generalist chewing insects (Holeski 2007a), and simulated damage addresses the effects of plant tissue loss that are independent of the effects of salivary compounds unique to particular herbivores (Inouye and Tiffin 2003). As evidence builds that some herbivores are able to avoid plant induction responses (Peiffer and Felton 2009), the separation of the effects of mechanical damage versus those of salivary compounds becomes increasingly important.

\section{The Genetic Architecture of Trichome Production}

We found a small number of QTLs with relatively large effects on trichome production in M. guttatus. Three aspects of trichome production - the probability of producing trichomes on second leaves, the probability of producing trichomes on fifth leaves, and constitutive trichome densitywere influenced to a large extent by the same genomic region. The QTL on LG 10 explained $10 \%-50 \%$ of the variation in the RIL population for each of these traits (table 3). Our selective genotyping method is specifically designed to find large-effect QTLs, but similar results have been obtained in trichome QTL studies of other plant species. For example, a single QTL was estimated to explain $43 \%$ of phenotypic variation in leaf trichome density in a cross between the cotton species Gossipium hirsutum and Gossipium barbadense (Wright et al. 1999; Lacape and Nguyen 2005). In Arabidopsis thaliana, a single QTL explained 73\% 
of the variation in first leaf-pair trichome density within an RIL population formed from a cross of plants from the Columbia and the Landsberg erecta ecotypes (Col-0 $\times$ Ler; Larkin et al. 1996). Symonds et al. (2005) mapped nine QTLs for fifth-leaf trichome density across four A. thaliana RIL populations, including a QTL overlapping the genomic region identified by Larkin et al. (1996). The relevance of these results to ODT is unclear, because trichomes serve a variety of functions and are not necessarily homologous structures in different species.

Pleiotropy is a key feature of genetic architecture and is the information that is most directly relevant to testing ODT. At our largest-effect QTL on LG 10, the Point Reyes allele increased the probability of producing trichomes on the second and fifth leaf pairs and increased constitutive trichome density. Correspondingly, we found significant, positive genetic correlations between the probabilities of producing trichomes on leaves of different developmental stages and between the probability of producing trichomes on a particular leaf pair and the density of trichomes produced. These results are similar to those of a mapping study in Arabidopsis, in which variation in constitutive trichome density on juvenile and adult leaves were influenced by a single major QTL (Mauricio 2005). The pleiotropic effects of the LG 10 and LG 14 QTLs suggest that the various trichome-related traits may have evolved in concert in ancestral populations, although here we caution that linkage disequilibrium among closely linked genes is an alternative explanation for the multiple phenotypic effects of a single QTL.

We found significant, negative genetic correlations between trichome 2 and induced trichome density and between constitutive trichome density and induced trichome density. These traits are all significantly influenced by the QTL on LG 14 (table 3), where the Point Reyes allele increased the probability of producing second-leaf trichomes and constitutive trichome density but reduced induced trichome density. In this case, the QTL-specific trait correlations are fully concordant with the overall genetic correlations. However, we note that the LG 14 QTL is the only mapped factor for induced trichome density, and it explains only $16 \%$ of the RIL variance. It is also possible that the effect of this QTL is contingent on genetic background. Epistasis is suggested by the nearly complete absence of trichome induction in the Iron Mountain parental line (Holeski 2007b), where the "high" allele (Iron Mountain) is homozygous. Instead, high induced trichome density was observed in RILs that are Iron Mountain homozygous at the LG 14 QTL but have a mixture of Iron Mountain and Point Reyes segments for the rest of the genome. We did not see any strong interaction between the QTLs of table 3 (using simple two-way ANOVAs), but our selective genotyping approach precluded a thorough scan for interactions across the genome.

The genetic architecture of trichome presence/absence was partially uncoupled from that governing trichome density. We identified two QTLs that significantly influenced only the probability of producing trichomes on a particular leaf pair (on LG 2 for trait trichome2 and on LG 5 for trait trichome5). The Point Reyes parental allele at the QTL on LG 2 decreased the probability of producing trichomes on the second leaf pair, while the Point Reyes allele at the QTL on LG 5 increased the probability of producing trichomes on the fifth leaf pair (table 3). Although these single-trait QTL explained only a small amount of the RIL-level population variation in trichome2 and trichome5, they indicate that some degree of independent evolution of these traits was possible in the natural ancestral populations of the RILs.

\section{Conclusions and Prospects}

The development of RILs from a cross between two divergent populations allowed us to elucidate novel patterns of genetic variation in the phenotypic plasticity and ontogeny of trichome production. There was generally good correspondence between observed genetic correlations and the results of QTL mapping for both ontogenetic and constitutive/induced trichome production. We documented a trade-off between constitutive and induced levels of defense at the QTL level. The direction of the trade-off corresponds to that predicted by ODT, given differential patterns of selection by herbivores and expected resource allocation priorities in coastal (Point Reyes) and alpine (Iron Mountain) populations.

Our genetic results motivate additional hypotheses about ODT that can be tested with field experiments. A reciprocal transplant experiment that includes the Iron Mountain $\times$ Point Reyes RILs (e.g., Hall et al. 2006) can estimate the reproductive fitness of RILs with high constitutive trichome density/low induced trichome density and vice versa in each environment. ODT predicts a reversal of selection across low- and high-herbivory habitats. Direct estimates for the current strength and direction of selection, both on individual traits and on trait combinations (e.g., constitutive and induced trichome densities), inform questions about the historical process of divergence in the multivariate trichome phenotype. Finally, we note that trichomes are only one of numerous herbivore defense traits in M. guttatus. The characterization of foliar phytochemistry and the chemistry of trichome glandular exudate (K. Keefover-Ring, L. M. Holeski, A. Clauss, D. Bowers, and R. Lindroth, unpublished data) will enable a broader range of hypothesis tests in the future. 


\section{Acknowledgments}

We thank J. Gleason, L. Hileman, S. Macdonald, M. Mort, and J. Ward, who generously shared their lab space/equipment for the genotyping portion of this study. Thanks to T. Marriage and J. Mojica, who helped with the DNA extractions, and to L. Fishman, Y. W. Lee, and J. Willis for their advice. J. Gleason, H. Holeski, K. Keefover-Ring, V. Koelling, S. Macdonald, A. Scoville, J. Ward, and two anonymous reviewers provided feedback that greatly improved previous drafts of this manuscript. This research was supported by National Institutes of Health grant GM073990 and National Science Foundation (NSF) grant DEB-0543052 (to J.K.K.). R. Lindroth provided financial support to L.M.H. (NSF grant FIBR-0425908) through the latter portion of this experiment and gave helpful comments on the manuscript.

\section{Literature Cited}

Agrawal, A. A. 2004. Plant defense and density dependence in the population growth of herbivores. American Naturalist 164:113120.

Agren, J., and D. W. Schemske. 1993. The cost of defense against herbivores: an experimental study of trichome production in Brassica rapa. American Naturalist 141:338-350.

Beardsley, P. M., and R. G. Olmstead. 2002. Redefining Phrymaceae: the placement of Mimulus, tribe Mimuleae, and Phryma. American Journal of Botany 89:1093-1102.

Berenbaum, M. R. 1981. Patterns of furanocoumarin production and insect herbivory in a population of wild parsnip (Pastinaca sativa L.). Oecologia (Berlin) 49:236-244.

Bergelson, J., and C. B. Purrington. 1996. Surveying patterns in the cost of resistance in plants. American Naturalist 148:536-558.

Butterworth, K. M., D. C. Adams, H. T. Horner, and J. F. Wendel. 2009. Initiation and early development of fiber in wild and cultivated cotton. International Journal of Plant Sciences 170:561574.

Churchill, G. A., and R. W. Doerge. 1994. Empirical threshold values for quantitative trait mapping. Genetics 138:963-971.

Darvasi, A. 1997. The effect of selective genotyping on QTL mapping accuracy. Mammalian Genome 8:67-68.

DeWitt, T. J., A. Sih, and D. S. Wilson. 1998. Costs and limits of phenotypic plasticity. Trends in Ecology \& Evolution 13:77-81.

Ehleringer, J. 1984. Ecology and ecophysiology of leaf pubescence in North American desert plants. Pages 113-132 in E. Rodrigues, P. L. Healey, and I. Mehta, eds. Biology and chemistry of plant trichomes. Plenum, New York.

Elle, E., and J. D. Hare. 2000. No benefit of glandular trichome production in natural populations of Datura wrightii? Oecologia (Berlin) 123:57-63.

Falconer, D. S., and T. F. C. Mackay. 1996. Introduction to quantitative genetics. 4th ed. Longmans Green, Harlow.

Feeny, P. 1975. Biochemical coevolution between plants and their insect herbivores. Pages 3-19 in L. E. Gilbert and P. H. Raven, eds. Coevolution of animals and plants. University of Texas Press, Austin.

1976. Plant apparency and chemical defense. Pages 1-40 in
J. W. Wallace and R. L. Mansell, eds. Recent advances in phytochemistry. Vol. 10. Plenum, New York.

Fenster, C. B., and K. Ritland. 1994. Quantitative genetics of mating system divergence in the yellow monkeyflower species complex. Heredity 73:422-435.

Fuller, W. A. 1987. Measurement error models. Wiley, New York.

Gravano, E., C. Tani, A. Bennici, and R. Gucci. 1998. The ultrastructure of glandular trichomes of Phillyrea latifolia L. (Oleaceae) leaves. Annals of Botany 81:327-335.

Hall, M. C., and J. H. Willis. 2005. Transmission ratio distortion in intraspecific hybrids of Mimulus guttatus: implications for genomic divergence. Genetics 170:375-386.

- 2006. Divergent selection on flowering time contributes to local adaptation in Mimulus guttatus populations. Evolution 60: 2466-2477.

Hall, M. C., C. J. Basten, and J. H. Willis. 2006. Pleiotropic quantitative trait loci contribute to population divergence in traits associated with life-history variation in Mimulus guttatus. Genetics 172:1829-1844.

Handley, R., B. Ekbom, and J. Agren. 2005. Variation in trichome density and resistance against a specialist insect herbivore in natural populations of Arabidopsis thaliana. Ecological Entomology 30:284-292.

Harvell, C. D., and R. Tollrian. 1999. Why inducible defenses? Pages 3-9 in R. Tollrian and C. D. Harvell, eds. The ecology and evolution of inducible defenses. Princeton University Press, Princeton, NJ.

Herms, D. A., and W. J. Mattson. 1992. The dilemma of plants: to grow or defend? Quarterly Review of Biology 67:283-335.

Holeski, L. M. 2007a. Quantitative trait evolution in Mimulus guttatus (yellow monkeyflower). PhD diss. Department of Ecology and Evolutionary Biology, University of Kansas, Lawrence.

-2007b. Within and between generation phenotypic plasticity in trichome density of Mimulus guttatus. Journal of Evolutionary Biology 20:2092-2100.

Holeski, L. M., and J. K. Kelly. 2006. Mating system and the evolution of quantitative traits: an experimental study of Mimulus guttatus. Evolution 60:711-723.

Inouye, B. D., and P. Tiffin. 2003. Measuring tolerance to herbivory with natural or imposed damage: a reply to Lehtila. Evolution 57: 681-682.

Iwata, H., and S. Ninomiya. 2006. AntMap: constructing genetic linkage maps using an ant colony optimization algorithm. Breeding Science 56:371-377.

Jordan, N. 1991. Multivariate analysis of selection in experimental populations derived from hybridization of two ecotypes of the annual plant Diodia teres W. (Rubiaceae). Evolution 45:1760-1772.

Karban, R., and I. T. Baldwin. 1997. How a plant perceives damage and signals other ramets, and the specificity of these processes. Pages 12-46 in R. Karban and I. T. Baldwin, eds. Induced responses to herbivory. University of Chicago Press, Chicago.

Karban, R., and J. S. Thaler. 1999. Phase change and resistance to herbivory. Ecology 80:510-517.

Karban, R., A. A. Agrawal, J. S. Thaler, and L. S. Adler. 1999. Induced plant responses and information content about risk of herbivory. Trends in Ecology \& Evolution 14:443-447.

Kelly, J. K. 2009. Connecting QTLs to the G-matrix of evolutionary quantitative genetics. Evolution 63:813-825.

Kelly, J. K., and H. S. Arathi. 2003. Inbreeding and the genetic variance in floral traits of Mimulus guttatus. Heredity 90:77-83.

Kliebenstein, D. J., A. Figureuth, and T. Mitchell-Olds. 2002. Genetic 
architecture of plastic methyl jasmonate responses in Arabidopsis thaliana. Genetics 161:1685-1696.

Lacape, J. M., and T. B. Nguyen. 2005. Mapping quantitative trait loci associated with leaf and stem pubescence in cotton. Journal of Heredity 96:441-444.

Larcher, W. 2001. Physiological plant ecology. Springer, Berlin.

Larkin, J. C., N. Young, M. Prigge, and M. D. Marks. 1996. The control of trichome spacing and number in Arabidopsis. Development 122:997-1005.

Lee, Y. W. 2009. Genetic analysis of standing variation for floral morphology and fitness components in a natural population of Mimulus guttatus (common monkeyflower). PhD diss. Duke University, Durham, NC.

Levin, D. A. 1973. The role of trichomes in plant defense. Quarterly Review of Biology 48:3-15.

Lexer, C., R. A. Randell, and L. H. Rieseberg. 2003. Experimental hybridization as a tool for studying selection in the wild. Ecology 84:1688-1699.

Lynch, M., and B. Walsh. 1998. Genetics and analysis of quantitative traits. Sinauer, Sunderland, MA.

Malakar, R., and W. M. Tingey. 2000. Glandular trichomes of Solanum berthaultii and its hybrids with potato deter oviposition and impair growth of potato tuber moth. Entomological Experiments and Applications 94:249-257.

Marquis, R. J. 1984. Leaf herbivores decrease fitness of a tropical plant. Science 226:537-539.

. 1992. The selective impact of herbivory. Pages 301-325 in R. S. Fritz and E. L. Simms, eds. Plant resistance to herbivores and pathogens: ecology, evolution, and genetics. University of Chicago Press, Chicago.

Mauricio, R. 1998. Costs of resistance to natural enemies in field populations of the annual plant Arabidopsis thaliana. American Naturalist 151:20-28.

. 2005. Ontogenetics of QTL: the genetic architecture of trichome density over time in Arabidopsis thaliana. Genetica 123:7585.

Mauricio, R., and M. D. Rausher. 1997. Experimental manipulation of putative selective agents provides evidence for the role of natural enemies in the evolution of plant defense. Evolution 51:1435-1444.

McKey, D. 1974. Adaptive patterns in alkaloid physiology. American Naturalist 108:305-320.

- 1979. The distribution of secondary compounds within plants. Pages 56-134 in G. A. Rosenthal and D. H. Janzen, eds. Herbivores: their interaction with secondary plant metabolites. Academic Press, Orlando, FL.

Medeiros, L., and G. R. P. Moreira. 2005. Larval feeding behavior of Gratiana spadicea (Coleoptera: Chrysomelidae: Cassidinae) on its host plant, Solanum sisymbrifolium (Solanaceae): interaction with trichomes. Coleopterists Bulletin 59:339-350.

Morris, W. F., M. B. Traw, and J. Bergelson. 2006. On testing for a tradeoff between constitutive and induced resistance. Oikos 112: 102-110.

Nagy, E. S. 1997. Selection for native characters in hybrids between two locally adapted plant subspecies. Evolution 51:1469-1480.

Orians, C. 2005. Herbivores, vascular pathways, and systemic induction: facts and artifacts. Journal of Chemical Ecology 31:22312242 .
Peiffer, M., and G. W. Felton. 2009. Do caterpillars secrete "oral secretions"? Journal of Chemical Ecology 35:326-335.

Poethig, R. S. 1997. Leaf morphogenesis in flowering plants. Plant Cell 9:1077-1087.

Purrington, C. B. 2000. Costs of resistance. Current Opinions in Plant Biology 3:305-308.

Rhoades, D. F. 1979. Evolution of plant chemical defense against herbivores. Pages 4-55 in G. A. Rosenthal and D. H. Janzen, eds. Herbivores: their interaction with secondary plant metabolites. Academic Press, Orlando, FL.

Ritland, K. 1989. Genetic differentiation, diversity, and inbreeding in the mountain monkeyflower (Mimulus caespitosus) of the Washington Cascades. Canadian Journal of Botany 67:2017-2024.

Romeis, J., T. G. Shanower, and A. J. Peter. 1999. Trichomes on Pigeonpea (Cajanus cajan (L.) Millsp) and two wild Cajanus spp. Crop Science 39:564-569.

Scoville, A., Y. W. Lee, J. H. Willis, and J. K. Kelly. 2009. Contribution of chromosomal polymorphisms to the G-matrix of Mimulus guttatus. New Phytologist 183:803-815.

Simms, E. L. 1992. Costs of plant resistance to herbivory. Pages 392 425 in R. S. Fritz and E. L. Simms, eds. Plant resistance to herbivores and pathogens: ecology, evolution, and genetics. University of Chicago Press, Chicago.

Simms, E. L., and M. D. Rausher. 1989. The evolution of resistance to herbivory in Ipomoea purpurea. II. Natural selection by insects and costs of resistance. Evolution 43:573-585.

Sokal, R. R., and F. J. Rohlf. 2000. Biometry. Freeman, New York.

Stamp, N. 2003. Out of the quagmire of plant defense hypotheses. Quarterly Review of Biology 78:23-55.

Strauss, S. Y., J. A. Rudgers, J. A. Lau, and R. E. Irwin. 2002. Direct and ecological costs of resistance to herbivory. Trends in Ecology \& Evolution 17:278-285.

Symonds, V. V., A. V. Godoy, T. Alconada, J. F. Botto, T. E. Juenger, J. J. Casal, and A. M. Lloyd. 2005. Mapping quantitative trait loci in multiple populations of Arabidopsis thaliana identifies natural allelic variation for trichome density. Genetics 169:1649-1658.

Traw, M. B., and P. Feeny. 2008. Glucosinolates and trichomes track tissue value in two sympatric mustards. Ecology 89:763-772.

van Dam, N. M., and J. D. Hare. 1998. Differences in distribution and performance of two sap-sucking herbivores on glandular and non-glandular Datura wrightii. Ecological Entomology 23:22-32.

Wang, S., C. J. Basten, P. Gaffney, and Z.-B. Zeng. 2007. Windows QTL Cartographer 2.0. Bioinformatics Research Center, North Carolina State University, Raleigh.

Werker, E. 2000. Trichome diversity and development. Advances in Botanical Research 31:1-35.

Wright, J. L., M. L. Stanton, and R. Scherson. 2006. Local adaptation to serpentine and non-serpentine soils in Collinsia sparsiflora. Evolutionary Ecology Research 8:1-21.

Wright R. J., P. M. Thaxton, K. M. El-Zik, and A. H. Paterson. 1999. Molecular mapping of genes affecting pubescence of cotton. Journal of Heredity 90:215-219. 